\title{
Levels of FGF23 predict outcomes in advanced CKD
}

Increased plasma levels of fibroblast growth factor 23 (FGF23) are associated with death, cardiovascular events and disease progression in patients with advanced chronic kidney disease (CKD) who are not yet on dialysis, according to a recent paper.

Increased serum levels of phosphate are known to be associated with an increased risk of death and cardiovascular events in patients with CKD not yet on dialysis and in those on dialysis. "Since the identification of FGF23, a phosphaturic hormone, there has been some interest in understanding the relationship between FGF23, the main hormone regulating phosphate balance, and important outcomes in patients with advanced CKD not requiring dialysis," explains Michel Chonchol, an author of the latest study. Raised levels of FGF23 have been shown to be associated with increased mortality in patients on dialysis and with disease progression in patients with mild CKD. However, whether levels of FGF23 are associated with death, cardiovascular events and dialysis initiation in patients with advanced CKD not yet on dialysis is unknown.

\section{4 ...FGF23 was a stronger predictor of outcomes than were traditional risk factors... 77}

Kendrick, Chonchol et al. analyzed stored blood samples from a cohort of 1,099 patients from the Homocysteine in Kidney and End Stage Renal Disease study. Measured factors included phosphorus, FGF23, parathyroid hormone and 1,25-dihydroxyvitamin D. Statistical analyses were carried out to determine whether levels of these factors were associated with adverse outcomes.
The researchers found that raised plasma levels of FGF23 were strongly associated with all-cause mortality, cardiovascular events and progression to chronic dialysis. This association was independent of other risk factors. In addition, FGF23 was a stronger predictor of outcomes than were traditional risk factors such as levels of vitamin D and parathyroid hormone. "This finding is important, as lots of resources have been directed to manage elevated parathyroid hormone levels when we should be more focused on phosphate balance," says Chonchol.

Plasma levels of FGF23 rise early in the course of kidney disease in order to maintain normal phosphorus levels. Thus, the authors suggest that plasma levels of FGF23 could potentially be used to guide therapy in patients with CKD. They also state that levels of FGF23 may be useful for identifying patients who might benefit from therapy to reduce their serum levels of phosphorus, even if they are within the normal range.

The mechanisms of the association between FGF23 and adverse outcomes are unknown. The authors speculate that FGF23 could have direct toxic effects on the cardiovascular system, or that the association is mediated or confounded by other factors. Studies are required to determine the mechanisms of the association and to investigate whether reducing levels of FGF23 will affect outcomes in patients with advanced CKD not yet on dialysis.

\section{Claire Greenhill}

Original article Kendrick, J. et al. FGF-23 associates with death, cardiovascular events, and initiation of chronic dialysis. J. Am. Soc. Nephrol. doi:10.1681/ ASN.2010121224 